EGU21-14058

https://doi.org/10.5194/egusphere-egu21-14058

EGU General Assembly 2021

(c) Author(s) 2022. This work is distributed under

the Creative Commons Attribution 4.0 License.

\title{
Temperature Dependent Entropy Driven Water Uptake in Phase Separated Aerosol from Steered Molecular Dynamics and Intrinsic Surface Analysis
}

Mária Lbadaoui-Darvas', Satoshi Takahama ${ }^{1}$, and Athanasios Nenes ${ }^{1,2}$

${ }^{1}$ Laboratory of Atmospheric Processes and their Impacts, ENAC, Ecole Polytechnique Fédérale de Lausanne, Lausanne, Switzerland

${ }^{2}$ Institute of Chemical Engineering Sciences, Foundation for Research and Technology Hellas, Patras, Greece

Dynamic water uptake by aerosol is a major driver of cloud droplet activation and growth. Interfacial mass transfer - that governs water uptake if the mean free path of molecules in the vapour phase is comparable to particle size - is represented in models by the mass accommodation coefficient. Although widely used, this approach neglects $i$ ) other internal interfaces (e.g., liquid-liquid that may be important for water uptake), and, ii) fluctuations of the liquid surface from capillary waves that modulate the surface and induce ambiguity in the estimation of mass accommodation coefficients. These issues can be addressed if the full path of the water molecule - from vapour to the bulk aqueous phase - is considered.

We demonstrate, using steered molecular simulations, that a full treatment of the water uptake process reveals important details of the mechanism. The simulations are used to reconstruct the free energy profile of water transport across a vapour/hydroxy cis-pinonic acid/water double interface at $300 \mathrm{~K}$ and $200 \mathrm{~K}$. In steered molecular dynamics the transferred molecule is pulled with a finite velocity along an aptly chosen reaction coordinate and the work exerted is used to reconstruct the free energy profile. Due to the finite velocity pulling, this method takes the effect of friction on the transport mechanism into account, which is important for phases of considerably different friction coefficients and is neglected by quasi equilibrium free energy methods. Free energy profiles are used to estimate surface and bulk uptake coefficients and are decomposed into entropic and enthalpic contributions.

Surface accommodation coefficients are unity at both temperatures, while bulk uptake at $300 \mathrm{~K}$ from the internal interface is strongly hindered $\left(k_{b}=0.05\right)$ by the increased density and molecular order in the first layer of the aqueous phase, which results in decreased orientational entropy. The difference between bulk and surface uptake coefficients also implies that water accumulates in the organic shell, which cannot be predicted using a single uptake coefficient for the whole particle. The minimum of the free energy profile at the organic/water interface, rationalised by increased conformational entropy due to local mixing and the depleted system density, results in a concentration gradient which helps maintain low surface tension and phase separation. Low surface tensions may explain increased CCN activity. These entropic features of the free energy 
profiles diminish at low temperature, which invokes a completely different mechanism of water uptake. Our results point out the need to describe water uptake in aerosol growth models using a temperature dependent parametrisation. 